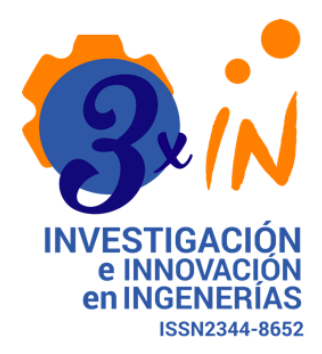

Open Access

Recibido:

10 de julio de 2019

Aceptado:

19 de noviembre de 2019

Publicado:

24 marzo de 2020

Correspondencia:

devinso_jimenez@cun.edu.co

DOI:

https://doi.org/10.17081/invinno.8.1 .3176

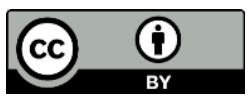

(c) Copyright: Investigación e Innovación en Ingenierías

\section{Mapa de actores de responsabilidad social empresarial en la ciudad de Santa Marta}

\author{
Map of corporate social responsibility actors in the city of Santa \\ Marta
}

\author{
Devinso José Jiménez Sierra (iD), Erica Cabrera (iD) \\ Antonio Ortega \\ Corporación Unificada Nacional - CUN, Colombia
}

Resumen

Objetivo: Mapear la dinámica de apropiación de planes y acciones de Responsabilidad Social Empresarial (RSE) en la ciudad de Santa Marta, evidenciando el uso del concepto asociado a RSE que podría generar una discusión en pro de mejorar las condiciones de la sociedad u entorno en el que se desarrollan las actividades empresariales. Metodología: En cuanto a la investigación es de tipo analítica, como herramienta principal para la construcción de la misma se utilizó el método MAC (Mapeo de Actores Claves) y a partir de ahí se estructura las diferentes líneas donde se reflejan los actores que intervienen en las variables establecidas. Resultados: Se describen la poca generación y disposición de las organizaciones públicas y privadas con alto impacto de gestión, se encontró la desconexión y baja articulación entre entidades que realizan actividades de impacto social afines, para la misma población desconociendo, ambos, sus intervenciones. Conclusiones: Se puede afirmar que la presente investigación muestra la dinámica que ejerce el sector empresarial en la ciudad de Santa Marta, analizado desde la Responsabilidad Social Empresarial (RSE), reconociendo la contribución de mejora que brindan a los diferentes escenarios (ambiental, educación, salud, entre otros) en los cuales se direccione la gestión de RSE. Entre los actores que sobresalen en la investigación hallamos organizaciones pertenecientes a diferentes sectores económicos, dándonos una panorámica del grado de responsabilidad social empresarial en la ciudad de Santa Marta.

Palabras claves: Responsabilidad social empresarial, mapa de actores, sociedad, entorno.

\begin{abstract}
Objective: Map the dynamics of appropriation of plans and actions of Corporate Social Responsibility (CSR) in the city of Santa Marta, evidencing the use of the concept associated with CSR that could generate a discussion in order to improve the conditions of the society or environment in the one that the business activities develop. Methodology: As for the research, it is of analytical type, as the main tool for the construction of the same, the MAC method (Key Actors Mapping) was used and from there the different lines are structured where the actors involved in the established variables. Results: The low generation and disposition of public and private organizations with high management impact are described, the disconnection and low articulation between entities that carry out related social impact activities were found, for the same population ignoring, both, their interventions. Conclusions: It can be affirmed that the present investigation shows the dynamics that the business sector exerts in the city of Santa Marta, analyzed from the Corporate Social Responsibility (CSR), recognizing the contribution of improvement that they give to the different scenarios (environmental, education, health, among others) in which CSR management is addressed. Among the actors that stand out in the research are organizations belonging to different economic sectors, giving us an overview of the degree of corporate social responsibility in the city of Santa Marta.
\end{abstract}

Keywords: Corporate social responsibility, map of actors, society, environment.

Como citar (IEEE): D. Jiménez, E. Cabrera., y A. Ortega, “Mapa de actores de responsabilidad social empresarial en la ciudad de Santa Marta", Investigación e Innovación en Ingenierías, vol. 8, n. 1, 2020. DOI: https://doi.org/10.17081/invinno.8.1.3176 


\section{Introducción}

En la presente investigación se realiza un análisis de la Responsabilidad Social Empresarial en la ciudad de Santa Marta desde la metodología MAC (Mapeo de Actores Claves), donde se muestran los niveles de influencia, rutas y relaciones entre organizaciones que realizan prácticas de RSE en la ciudad de Santa Marta. El estudio se realiza contemplando los diferentes escenarios empresariales activos en la ciudad; entidades gubernamentales, no gubernamentales y organizaciones del sector privado. En una primera fase se monitorearon empresas reconocidas por sus actividades de impacto social. En una segunda fase se creó un cuadro de variables cuyos ítems abordan aspectos con relación a las estrategias empresariales definidas para ejecutar acciones de RSE, se contemplan los diferentes proyectos ejecutados por las organizaciones e igualmente se corrobora su visibilidad, en una fase final se mapeo las relaciones y acciones para establecer canales de comunicación y rutas de actores de RSE de la ciudad de Santa Marta. Asimismo se muestran las relaciones establecidas para formalizar los diferentes convenios o ayudas, los cuales permiten la puesta en marcha del proyecto y por último se indaga de cuales empresas tienen certificados de RSE.

\section{Fundamento teórico}

\section{Percepción de la RSE en el ámbito empresarial actual}

La globalización ha traído nuevos conceptos para la construcción de metodologías que propicien una mejor toma de decisiones, Las empresas se mueven en un escenario de relevancia tanto económica como política debido al rol que juegan en sectores estratégicos de la economía y su influencia en el ámbito público [1]. Pero estas no están dimensionando todo la influencia que generan y sólo se concentran en factores económicos, olvidando su entorno, acotando las brechas que nos acercan a la pobreza, las desigualdades sociales; los escándalos contables, las quiebras fraudulentas, la corrupción, las adquisiciones ilícitas, la desconexión entre las actividades financieras y económicas, la violación de los derechos laborales y el deterioro ambiental.

Desde esta perspectiva nace el concepto de la responsabilidad social empresarial, de aquí en adelante (RSE), con el fin de generar una estabilidad entre lo obligatorio y lo justo; contribuir en mejorar las condiciones de la sociedad en donde todos ganamos. La RSE se define como el compromiso que las empresas deben tener respecto de sus acciones que involucran a empleados, la sociedad y al medio ambiente, teniendo un comportamiento integro con el fin de aplicar medidas que vaya en pro de satisfacer las necesidades de todos [1]. 
Mientras que el término RSE puede parecer relativamente nuevo en el mundo de la empresa, la literatura revela que la evolución del concepto en sí ha tenido lugar durante varias décadas, la mayoría de la investigación empírica sobre RSE se ha concentrado en el ámbito de las grandes empresas [2]. Pasó de ser un simple concepto a convertirse en un método que tienen las empresas para gestionar, independientemente a su tamaño u razón social, esto permite ampliar la capacidad de comprender sus actividades diarias desde una perspectiva sostenible. La RSE contribuye positivamente a las empresas, por lo que estas se han visto obligadas a entender y analizar, que está sucediendo actualmente sobre este tema, e intervenir sus políticas éticas, económicas, y ambientales, para una toma de decisión justa que contribuyan a una sociedad más solidaria.

La empresa moderna asume un reto histórico en la construcción de sentido de los procesos de cohesión social, para ello el concepto de RSE se erige como un instrumento de tránsito que nos acerca a la construcción de una sociedad con más equidad y justicia [3]. Esta tendencia, desde la sostenibilidad, es observada por los gobiernos, investigadores y organismos con relación a su compromiso con el cuidado del medio ambiente, el apoyo a la sociedad y a su desarrollo económico, por lo cual, las empresas deben implementar estrategias éticas y responsables con sus diferentes grupos de interés: socios, capital humano, clientes, comunidad, proveedores, autoridades gubernamentales y organismos [4].

En Colombia aún no se ha implementado una política que obliguen a las empresas, desde la aplicación de una ley, que la regule y generen estándares mayormente vinculantes a la RSE. En estos roles, los gobiernos y las administraciones públicas tienen un papel fundamental, difícilmente sustituible. "Por lo cual, las empresas deben implementar estrategias éticas y responsables con sus diferentes grupos de interés: socios, capital humano, clientes, comunidad, proveedores, autoridades gubernamentales y organismos" [4]. Todo ello hace que sea cada vez más necesario que las empresas y los países tengan una clara conciencia de la necesidad de elaborar su propia aproximación a la RSE. Esto hace que la RSE no se refiera ya simplemente a las relaciones entre empresa y sociedad, sino que se configure como una manera de repensar el papel de la empresa en la sociedad, incorporando como elemento vertebrador una perspectiva de gobernanza y sostenibilidad [5].

Hoy en día las empresas utilizan el denominado marketing social con el propósito de obtener un beneficio económico como los genera el marketing comercial, sin tener en cuenta que en realidad los criterios de sostenibilidad son otros, aprovechándose de la falta de conocimientos de este concepto, por esta razón este tipo de marketing es visto como un engaño a la sociedad [6]. Además Es preciso saber que las empresas se aprovechan y utilizan la RSE como estrategia, para cubrir las faltas que causan al desarrollar sus actividades productivas. Según las empresas han presentado dichas acciones 
como responsabilidad social empresarial, olvidando o desconociendo convenientemente que la reparación del daño es una obligación moral y resultado automático de una culpa moral" [7].

Por otro lado; la construcción de mapas no es una actividad nueva en las ciencias sociales, la cartografía social responde a una visión de la realidad desde la geografía del territorio [8]. Todo proceso de descubrimiento está asociado a la activad geografía, esto permite reconocer limites, conexiones, cruces y formas en que se estructura un espacio social [9]. Los mapas son representaciones, con cargas dialógicas que permiten interpretaciones y una semántica distinta a la forma precisa de lenguaje formal. Según Herley [9] el mapa, como elemento intertextual, posee una función de referencia y adecuación sobre la espacialidad y la vivencia, que en algunos momentos se configura como histórica, también.

Al referirnos al mapeo de actores, responde a la manera en que una entidad organizacional o de representación, actúa en un determinado lugar, permite identificar la existencia de conexiones, relaciones o cruces entre organizaciones que propenden un mismo fin, en este caso conocer las acciones asociadas a RSE. Para Gadamer [10]; existe un sentido del espacio en el que el sujeto, ente cognoscente, y el objeto, fuente de conocimiento, se funden en un horizonte que él denomina horizonte dialógico; conversación entre la representación del espacio y las relaciones del espacio.

La representación gráfica del espacio, por medio de los MAC permite el reconocimiento de las dinámicas sociales desde la identificación de espacios vividos más allá de la tradicional intertextualidad del documento. Se asocian con otras imágenes y permiten realizar lecturas de tipo relacional teniendo como horizonte la ubicación cartográfica, en palabras de Lindón; espacio abstracto con espacio vivencial. Los mapeos sociales generan identidad con el espacio, puesto que crea identificación con lugares que se recrean en la memoria [11]. '‘Lo vivido' a los límites de 'lo visible', es el resultado de una combinación entre una visión de poder, a través de la institucionalización, producción y difusión de un espacio abstracto" [12]. Al estudiar el espacio y su representación geográfica desde sus elementos historiográficos y su particular forma de generar discurso se potencializa como dadora de significado e imaginarios geográficos. El saber geográfico es una perspectiva particular de configurar el espacio.

\section{Metodología}

El mapeo de actores de responsabilidad social empresarial en la ciudad de Santa Marta responde a un enfoque hermenéutico de carácter descriptivo entendido como construcción de la realidad empírica desde la 
interpretación taxonómica de los datos [13], de enfoque positivista, al respecto Vargas, dice que "el positivismo, es la adopción de una noción limitada del método científico no sólo como una prescripción para desarrollar investigación y producir conocimiento, sino como una visión comprehensiva, una ideología social y una definición del significado de la vida." [13]. De esto se deriva que esta investigación responde a una realidad empírica en el contexto social de las entidades gubernamentales y privadas de la ciudad de Santa Marta.

En relación con el diseño de la investigación, se observaron y describieron las categorías asociadas a la Responsabilidad Social Empresarial; a saber, los planes de RSE, las estrategias, su visibilidad, los proyectos en ejecución, los convenios asociadas a las entidades objetos de estudio y su condición de certificadas en RSE. Es transversal, al respecto Hernández, Fernández y Baptista [14] consideran que al "recolectar datos en un solo momento, en un tiempo único, su propósito es describir categorías y analizar su incidencia e interrelación en un momento dado" (p. 9).

\section{Población y Muestra}

La población está constituida por entidades gubernamentales y privadas de la ciudad de Santa Marta. La muestra es no probabilística de tipo discrecional; este tipo de muestra se selecciona desde el juicio de un experto o condición especial acerca de la contribución que las unidades de muestreo harán para responder a la pregunta de investigación [15]. Para esta investigación corresponde a los entes gubernamentales o privados de la ciudad de Santa Marta que a la fecha del estudio tenía constituidas áreas y/o departamentos para la gestión de la Responsabilidad social, lo que arrojo una relación de siete entidades gubernamentales y trece empresas privadas.

\section{Técnicas para la recolección de información}

El estudio se desarrolló desde dos elementos que juntos constituyen un Mapeo de Actores Claves (MAC); primero, una matriz de involucrados tomado de metodología de marco lógico. Segundo, una matriz de fuerza entre poder (P) e Influencia (I) de las organizaciones objeto de estudio. La metodología MAC, es un modo de representación de la realidad en contexto social, no sólo para identificar posibles actores sino para reconocer las actividades y objetivos de la organización en función del territorio [16] Además, el MAC es una herramienta ampliamente vinculada a los estudios de redes sociales con enfoque cualitativo en el que los actores se caracterizan desde su interacción y conectividad entre organizaciones dentro de un mismo escenario [17]. Abordad la realidad social desde la perspectiva de redes implica el cruce de información y las relaciones vinculantes entre categorías afines desde diferentes actores; de este modo, estás interacciones generan distintas actuaciones que condicionan sus valores, creencias y comportamientos. 


\section{Instrumentos}

La matriz de involucrados está construida para identificar los elementos propios para determinar acciones de responsabilidad social empresarial; se describieron los planes públicos con socialización de resultados; las estrategias para las mismas; su impacto de visibilidad identificando sus canales de divulgación, distintos a la socialización publica de los planes; describiendo los proyectos en desarrollo; indagando por medio de entrevistas a funcionarios, los convenios o ayudas que brindaban en temas de responsabilidad social Empresarial; por último, verificando su condición de certificados por alguna entidad con esta competencia en el área específica de la RSE (ver Tabla 1).

Tabla 1. Acciones de RSE de entidades gubernamentales y privadas

\begin{tabular}{|c|c|c|c|c|c|c|}
\hline $\begin{array}{c}\text { Número de } \\
\text { entidades }\end{array}$ & $\begin{array}{c}\text { Planes } \\
\text { RSE }\end{array}$ & $\begin{array}{c}\text { Estrategias de } \\
\text { RSE }\end{array}$ & Visibilidad & $\begin{array}{c}\text { Proyectos } \\
\text { desarrollados }\end{array}$ & $\begin{array}{c}\text { Convenios o } \\
\text { ayudas }\end{array}$ & $\begin{array}{c}\text { Certificado de } \\
\text { RSE }\end{array}$ \\
\hline
\end{tabular}

Fuente: Elaboración propia

En la matriz de fuerza entre poder $(P)$ e influencia $(I)$ se presenta los criterios definidos para valorar las dimensiones de poder e interés de cada uno de los actores identificados con relación a los temas de diseño e implementación de la matriz de involucrados. El poder y la influencia de los actores fueron identificados por medio de formularios paralelos utilizados en la Mesa de Discusión y Análisis utilizando la metodología Delphos en la que se asignaron valores ente $[0,1-1]$ según la condición de cada actor (ver Tabla 2).

Tabla 2. Acciones de RSE de entidades gubernamentales y privadas

\begin{tabular}{|c|c|c|c|}
\hline Condición & Poder & Influencia & Acción R \\
\hline Activa & 1 & 1 & \multirow{2}{*}{ xR/P*R } \\
\hline Pasiva & 0,6 & 0,6 & \\
\hline Indeciso & 0,3 & 0,3 \\
\hline Desconocida & 0,1 & 0,1 & \\
\hline
\end{tabular}

Fuente: Elaboración propia

Con el propósito de obtener el criterio de validez de la escala utilizada se solicitó la participación de tres expertos, observando la pertinencia de las categorías asignadas para la matriz de involucrados y la matriz de fuerza (P) vs (I) También se entregó un formato de validación, copia de las matrices, para que emitieran sus juicios, consideraciones en cuanto al contenido. Al valorar los instrumentos el índice de validación fue de 0,68 en una escala de cero a uno, generada como media aritmética de las valoraciones de los expertos sobre las categorías de la medición. 


\section{Resultados y discusión}

Al enlistar las entidades objeto de estudios identificamos que sólo siete entidades gubernamentales cuentan con un área o departamento o sección dedicada a la gestión de la RSE frente a trece empresas del sector privado. Al resumir sus planes encontramos que las Entidades Gubernamentales, en adelante (EG) tiene siete planes de RSE, uno por cada entidad, frente a los ocho planes que definieron las Empresas Privadas, en adelante (EP). Al indagar sobre las acciones de RSE se presentan siete por parte de las EG y diecisiete las EP para un total de veinticuatro acciones de RSE, de las cuales sólo nueve tiene divulgación. Las EP tienen más proyectos en desarrollo (21) en total frente a sólo seis por parte de EG. Las EG presentan ocho convenios asociados a RSE frente a seis por parte de EP. Frente a procesos de certificación en RSE ninguna EG lo ha hecho frente a cinco certificadas en EP. (Ver Tabla 3).

Tabla 3. Acciones de RSE de entidades gubernamentales y privadas

\begin{tabular}{|c|c|c|c|c|c|c|c|}
\hline & $\begin{array}{c}\text { Número } \\
\text { de } \\
\text { entidades }\end{array}$ & $\begin{array}{c}\text { Planes } \\
\text { RSE }\end{array}$ & $\begin{array}{c}\text { Estrategias de } \\
\text { RSE }\end{array}$ & Visibilidad & $\begin{array}{c}\text { Proyectos } \\
\text { desarrollados }\end{array}$ & $\begin{array}{c}\text { Convenios } \\
\text { o ayudas }\end{array}$ & $\begin{array}{c}\text { Certificado } \\
\text { de RSE }\end{array}$ \\
\hline $\begin{array}{c}\text { Entidades } \\
\text { gubernamentales }\end{array}$ & 7 & 7 & 7 & 5 & 6 & 8 & 0 \\
\hline $\begin{array}{c}\text { Empresas del } \\
\text { sector privado }\end{array}$ & 9 & 5 & 7 & 4 & 21 & 6 & 5 \\
\hline
\end{tabular}

Fuente: Elaboración propia

Al revisar las declaraciones de RSE de las organizaciones objeto de estudio encontramos que en las entidades gubernamentales se desarrollan siente planes de RSE, básicamente en dos ejes, desde los compromisos ambientales y los compromisos sociales con las comunidades circundantes, en particular con procesos asociados a la educación. Por parte de las entidades privadas objeto de estudio, encontramos ocho planes de RSE, con el mismo énfasis de lo social y ambiental sin discriminar específicamente algún grupo de interés (ver Tabla 4). 
Tabla 4. planes de RSE de entidades gubernamentales y privadas

\begin{tabular}{|c|c|}
\hline & Planes RSE \\
\hline 1 & Entidades Gubernamentales \\
\hline 2 & Sostenibilidad y protección ambiental \\
\hline 3 & Concejo de política social \\
\hline 4 & Problemática ambiental \\
\hline 5 & Sostenimiento del medio ambiente \\
\hline 6 & Educación gratis y estructura social \\
\hline 7 & El modelo de RSE de ECOPETROL parte de la definición de un compromiso explícito de relacionamiento con cada \\
\hline & grupo de interés. \\
\hline 1 & Empresas Privadas \\
\hline 2 & Está orientado a satisfacer las necesidades y expectativas de los grupos de interés. \\
\hline 3 & Desarrollo comunitario \\
\hline 4 & Plan de gestión social \\
\hline 5 & Protección a la biodiversidad \\
\hline 6 & Comunidades sostenibles \\
\hline 7 & Educación, desarrollo del capital social, generación de ingresos \\
\hline 8 & Apoyo a la educación \\
\hline
\end{tabular}

Fuente: Elaboración propia

Al discriminar las acciones de RSE encontramos para las entidades gubernamentales el mismo número de acciones para igual número de planes con amplio énfasis en el eje medio ambiental dirigido a una población específica como lo son la niñez y la adolescencia. Las diferentes acciones tienen como característica; procesos de formación en preservación del medio ambiente, pero no intrínsecas al fenómeno de la RSE. Por parte de las entidades privadas el número de acciones duplica el número de planes, en las actividades se amplía el espectro de acción, incluyendo acciones asociadas a desarrollo laboral, promoción de voluntariados, reconocimientos públicos, entre otros (ver Tabla 5).

Tabla 5. Acciones de RSE de entidades gubernamentales y privadas

\begin{tabular}{|c|c|}
\hline & Acciones de RSE \\
\hline & Entidades Gubernamentales \\
\hline 1 & Siembra de árboles y arbustos en distintos sectores de la ciudad \\
\hline 2 & Movilización social por una educación de calidad \\
\hline 3 & $\begin{array}{l}\text { Inclusión social con de la niñez y adolescencia, reconociéndolos como presente activo y Participativo para } \\
\text { generar cambio social en el desarrollo del distrito. }\end{array}$ \\
\hline 4 & Implementación de grupos para garantizar la atención oportuna \\
\hline 5 & Siembra de árboles nativos y protección por el medio ambiente \\
\hline 6 & Formación de aprendizaje en diferentes áreas, inclusión de grupos de personas \\
\hline \multirow[t]{2}{*}{7} & $\begin{array}{l}\text { Asegurar la sostenibilidad del negocio, construir un gran lugar para trabajar, asegurar la satisfacción del cliente, } \\
\text { contribuir a la construcción de un estado social, participar en el desarrollo sostenible del país. }\end{array}$ \\
\hline & Empresas Privadas \\
\hline 1 & $\begin{array}{l}\text { Gestionar proyectos sostenibles direccionados al mejoramiento de calidad de vida, educación, preservación del } \\
\text { medio ambiente y su entorno. }\end{array}$ \\
\hline
\end{tabular}




\begin{tabular}{|c|c|}
\hline 2 & $\begin{array}{c}\text { Construcción de escuelas, patrocinio de becas universitarias, establecimientos de centros de salud, financiación } \\
\text { de centros de capacitación laboral. }\end{array}$ \\
\hline 3 & Promover el reconocimiento de los distintos tipos de conductas intimidadoras. \\
\hline 4 & $\begin{array}{c}\text { Fortalecimiento de capacidades y empoderamiento de personas, su gestión se centra en aspectos como la salud, } \\
\text { educación y atención a la comunidad. }\end{array}$ \\
\hline 5 & $\begin{array}{c}\text { Formar a los estudiantes a través de proyectos de aprendizaje desde el servicio voluntario, promoviendo valores } \\
\text { por todos los seres vivos. }\end{array}$ \\
\hline 6 & Formación en producción agrícola y orgánica a jóvenes. \\
\hline 7 & Apoyar la transformación y enriquecimiento de los programas de formación de instituciones oficiales. \\
\hline 8 & Formar a las mujeres en la producción de hortalizas y aromáticas. \\
\hline 9 & Mejoramiento del 100\% de las instituciones educativas del área de influencia directa. \\
\hline 10 & Programas para el desarrollo del capital social. \\
\hline 12 & Becas a niños y jóvenes de escasos recursos \\
\hline 13 & Apoyos a madres cabeza de familia \\
\hline 14 & Medio ambiente \\
\hline 15 & premio mujer palmera campesina \\
\hline 16 & Alianzas estratégicas (red colaborativa) \\
\hline 17 & Pramas especiales \\
\hline
\end{tabular}

Fuente: Elaboración propia

Al aplicar la matriz de fuerza poder (vs) Influencia desde la valoración ponderada de los tres jueces expertos; según la percepción sobre las organizaciones objeto de estudio, se evidenció que sólo tres entidades tienen alto poder y alta influencia. Ocho de las veinte entidades tiene poder medio e influencia media, mientras que las nueve entidades restantes tiene bajo poder y baja influencia. Hay que aclarar que las acciones de RSE descritas desde los planes de cada entidad responden a procesos internos, no se evidencia articulación o gestión de los axiomas ligados a RSE. Es decir a pesar de que las entidades gubernamentales ejercen actividades asociadas a RSE, estas no tienen por fin fijar políticas para que otras entidades o sectores practiquen la RSE. Esto se evidencia en el cruce de fuerzas (ver Tabla 6, Figura 1).

Tabla 6. Acciones de RSE de entidades gubernamentales y privadas

\begin{tabular}{|c|c|c|c|c|c|c|c|c|c|c|c|c|c|c|c|c|c|c|c|c|}
\hline Entida & $\mathrm{e}$ & $\mathrm{e}$ & $\mathrm{e}$ & $\mathrm{e}$ & $\mathrm{e}$ & $\mathrm{e}$ & $\mathrm{e}$ & $\mathrm{e}$ & $\mathrm{e}$ & $\mathrm{e}$ & $\mathrm{e}$ & $\mathrm{e}$ & $\mathrm{e}$ & $\mathrm{e}$ & $\mathrm{e}$ & $\mathrm{e}$ & $\mathrm{e}$ & $\mathrm{e}$ & $\mathrm{e}$ & $\mathrm{e}$ \\
des & 1 & 2 & 3 & 4 & 5 & 6 & 7 & 8 & 9 & 1 & 1 & 1 & 1 & 1 & 1 & 1 & 1 & 1 & 1 & 2 \\
& & & & & & & & & & 0 & 1 & 2 & 3 & 4 & 5 & 6 & 7 & 8 & 9 & 0 \\
\hline cruce & 0, & 0, & 0, & 0, & 0, & 0 & 0, & 0, & 0, & 0, & 0, & 0, & 0 & 0 & 0, & 0, & 0, & 1 & 1 & 0 \\
P vs I & 1 & 2 & 3 & 2 & 3 & 2 & 3 & 1 & 2 & 6 & 5 & 5 & 5 & 6 & 5 & 5 & 6 & & & 9 \\
\hline
\end{tabular}

Fuente: Elaboración propia 
Figura 1. Resultado matriz de fuerzas P vs I: La figura muestra la posición de las entidades según su nivel de poder e influencia como resultado de la matriz de fuerza

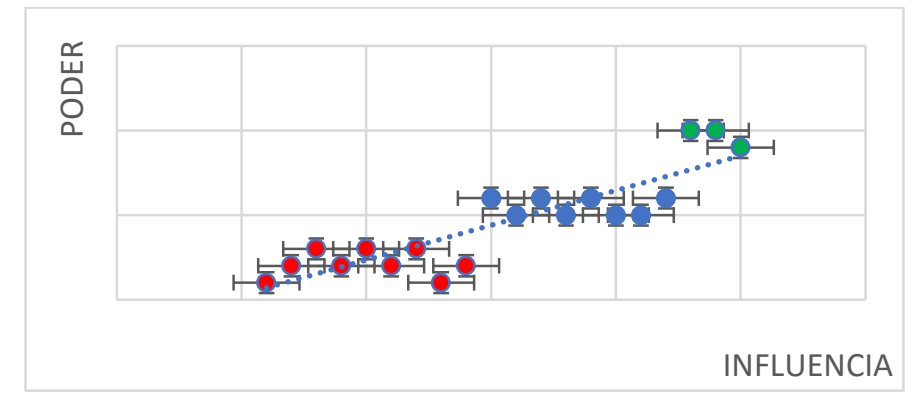

Fuente: Elaboración propia

En la Figura 2, se muestra la construcción de una red semántica, con la valoración de los jueces expertos, en la que se expone la relación de poder e influencia de las entidades objeto de estudio, en la ciudad; esto evidenció que existe una entidad gubernamental, del orden distrital, y dos entidades privadas que tienen alto poder y alta influencia; esto quiere decir que cuentan con posicionamiento en la ciudad para desarrollar proceso de gestión en red de acciones de RSE con impacto en la ciudad, pero que aún no ejercen ese poder e influencia de manera proyectual.

En un segundo grupo encontramos ocho entidades, cuatro del orden privado y cuatro del orden público, con alto poder en el sector en el que se desempeñan y bajo nivel de influencia; esto significa que estas organizaciones tienen posibilidades de desarrollar vínculos inter-gremiales en sus respectivos sectores pero con poco relacionamiento para el desarrollo de las RSE. En el último segmento encontramos nueve entidades, dos del orden público y siente del orden privado con bajo poder y baja influencia; entidades que desarrollan planes y acciones de RSE pero sin impacto intergremial, ni relacionamiento interempresarial.

Con la información recopilada en las matrices y las valoraciones de los jueces expertos se diseñó un mapa con las rutas de interconexión existente entre entidades públicas y privadas (ver Figura 3), las rutas están demarcadas por colores y representan las áreas afines en las que trabajan pero sin fijar actividades asociadas, es decir que varias de estas entidades tiene proyectos afines pero esfuerzos por separado; en el tema de la educación encontramos la relación de cuatro entidades con acciones en RSE afines, planes de mejoramiento continuo en educación, consejo de política social, con componente educacional y proyectos de formación en educación sostenible en el que colegios públicos son los mayores beneficiarios. Otro caso de concordancia es la preocupación por escenarios de post-acuerdo, en el que 
varias entidades tanto públicas como privadas concuerdan pero desde acciones simplificadas y excluyentes.

Las Rutas de RSE de la ciudad de Santa Marta muestran las debilidades que existen en la articulación mancomunadas de las organizaciones públicas y privadas que en el ejercicio de sus ideales corporativos desarrollan acciones, planes de RSE sin ningún tipo de articulación formal lo que desestima los resultados a impactos particulares y no a una política social para la promoción de valores asociados a RSE.

Figura 2. Red semántica: relación poder vs Influencia.

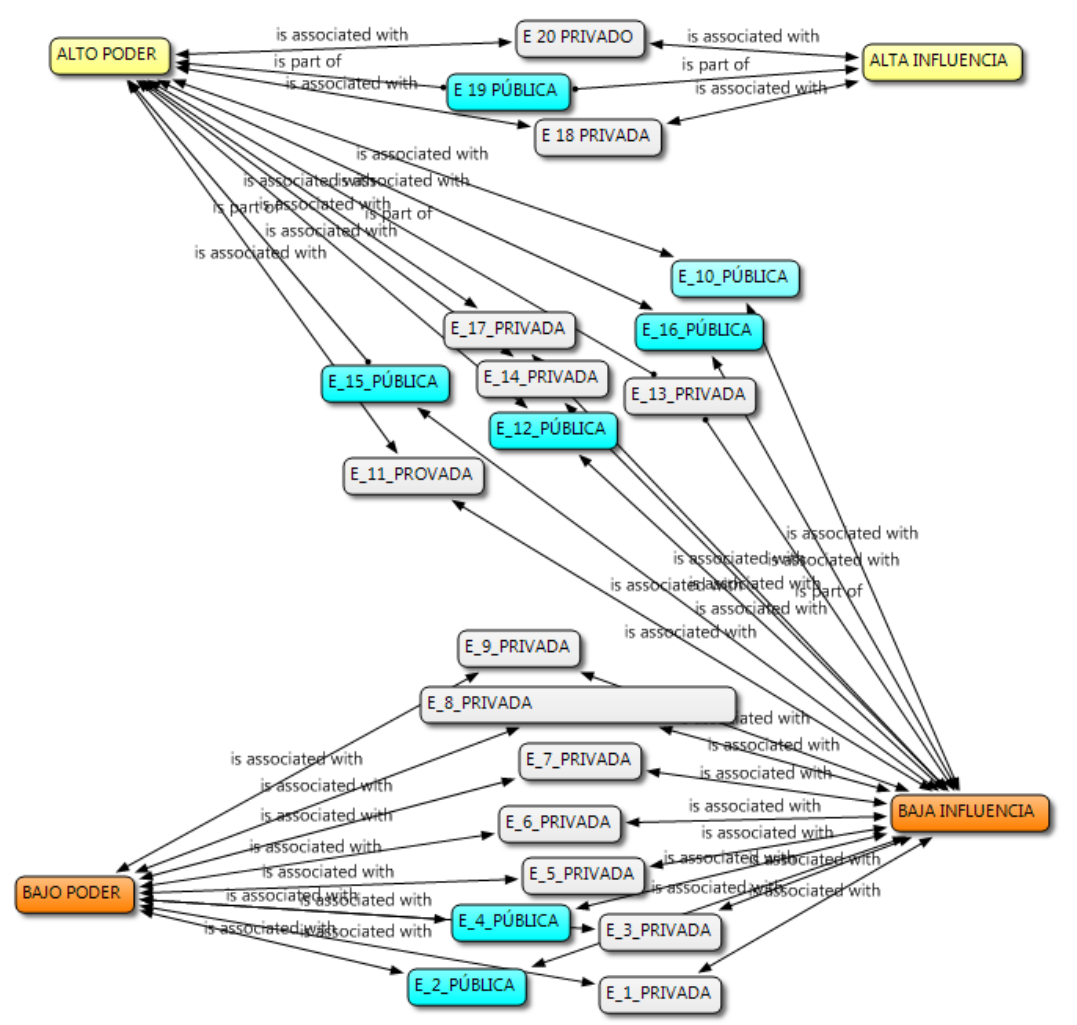

Fuente: Elaboración propia

La red semántica es una herramienta hermenéutica que permite realizar asociaciones entre categorías. Aquí se muestran las interacciones de las entidades privadas y públicas que gestionan RSE en la ciudad de Santa Marta en relación a su poder e influencia según las calificaciones de tres jueces expertos. Construcción a partir de Atlas -ti V. 7. 
Figura 3. Ruta de acciones de RSE en Santa Marta

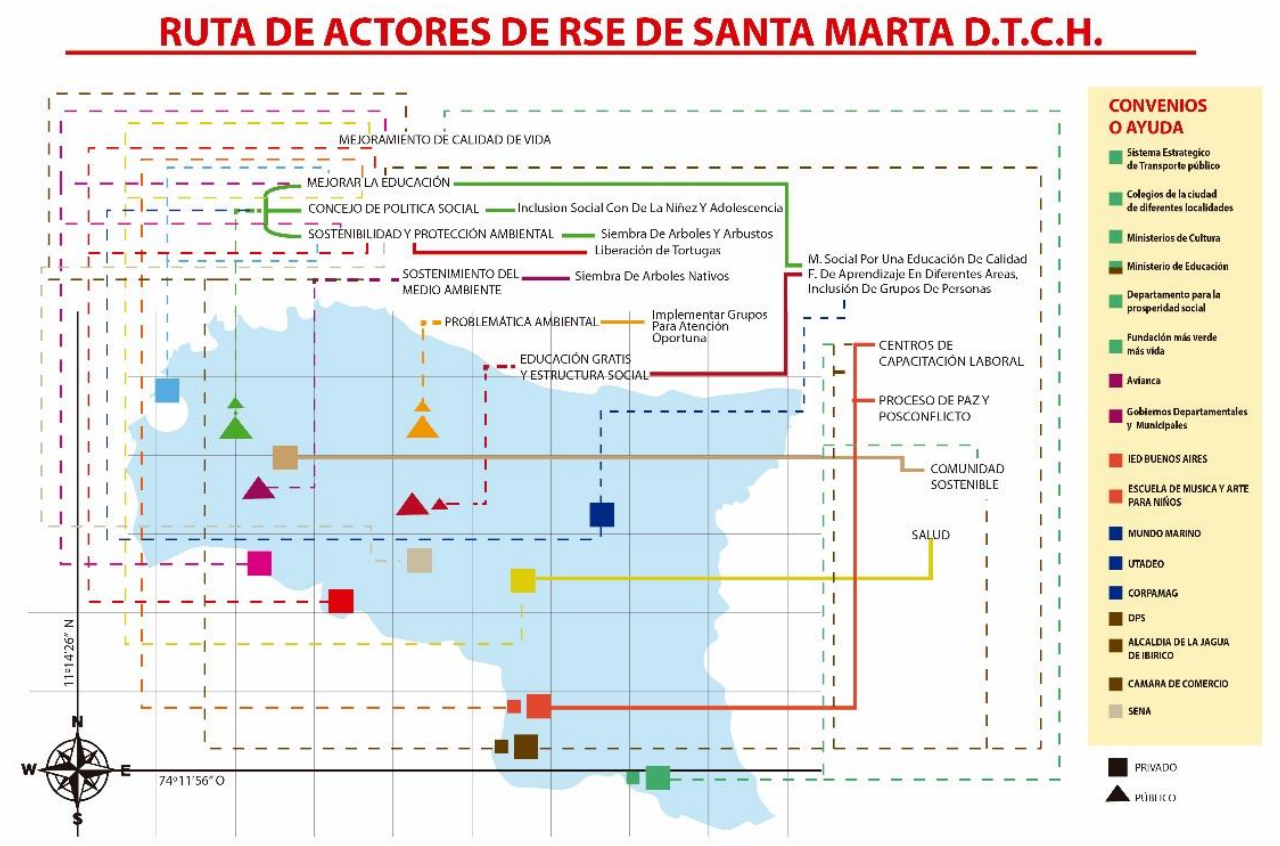

Fuente: Elaboración propia

La Figura 3 muestra las acciones que tienen intereses comunes, áreas similares de trabajo, planes de RSE que podrían ser vinculantes, estás están demarcadas por líneas punteadas que se enlazan por medio de colores (La construcción es propia desde un software de diseño Corel Draw v. 15). Los triángulos representan entidades públicas y los cuadrados entidades privadas.

\section{Discusión}

En la ciudad de Santa Marta no se identifican actores de la RSE interrelacionados en procesos de construcción de redes visibles e incluyentes, lo que condiciona la apropiación de los conceptos básicos asociados la RSE. La cartografía generada por medio de la investigación muestra la poca gestión de los entes gubernamentales y privados para involucrar, sensibilizar y cohesionar actividades, acciones o planes de RSE como lenguaje de participación social, a pesar de contar con el posicionamiento estratégico y la influencia para gestarlo.

Los autores coinciden qué la RSE es un instrumento gerencial muy importante y necesario, porque logra que las empresas generen estrategias que les faciliten su evolución y se ajusten a las condiciones del entorno. En este sentido, la RSE ha sido propuesta en los últimos tiempos como una de las estrategias más efectivas para diferenciarse y fortalecer la competitividad de las Pymes [18] Las entidades gubernamentales no tienen 
estructurado planes de gestión que asocien, agrupen, conecten o visibilicen las prácticas de RSE en la ciudad. Son las entidades privadas las que están más cerca de conectarse entre sí, aquí juega un papel importante las entidades que promueven procesos de certificación, en particular FENALCO solidario, quien constantemente promueve valores de RSE en la ciudad pero con poco impacto. Los entes gubernamentales deben no solo integrar los principios de responsabilidad a sus propios sistemas de gestión, además deben proyectar las interacciones con sus interlocutores desde la posición del servicio social que representan. L a discusión actual se centra en el ejercicio del poder que han de desempeñar los entes gubernamentales, la capacidad de difusión y gestión de la RSE desde una posición formal que contribuya a una cultura corporativa responsable y social, entendiendo que puede favorecer las ventajas competitivas de las empresas en sus determinados mercados [19].

\section{Conclusiones}

Como primera conclusión podemos afirmar que la RSE en la ciudad de Santa Marta está representada en los planes y acciones de las entidades gubernamentales y privadas con más poder e influencia de la ciudad, a su vez esta perspectiva es meramente intrínseca a las mismas organizaciones, sin que existan planes de promoción de la RSE como herramienta de cohesión social y/o estrategia de desarrollo sostenible.

En una siguiente conclusión, la investigación muestra que estas acciones intrínsecas no están relacionadas ni interconectadas a pesar de contar con temas afines, áreas de desarrollo afines e incluso poblaciones beneficiarías similares, esto muestra la desarticulación que existe a nivel gremial y gubernamental entorno a la RSE. Aún más si se estima que los entes gubernamentales desde su estructura de entidad social no propenden estrategias para la construcción de diálogos que permitan incorporar al sistema primario de comunicación los conceptos asociados a RSE.

Como conclusión final encontramos que a pesar que, según la valoración de los jueces expertos, las tres entidades con mayor poder e influencia, donde se instala una del orden gubernamental, no presentas estrategias desde las políticas públicas, ni desde acciones de relacionamiento una preocupación por extender al pleno de las organizaciones de la ciudad un escenario de discusión de la RSE como herramienta de desarrollo sostenible acorde con los indicadores de competitividad local, regional y nacional. 


\section{Referencias bibliográficas}

1. M. García., y J. Gerardo, "La responsabilidad social empresarial en el contexto del capital social", Omnia, pp. 46-59., 2016.

2. J.H. Madueño, M. Larrá., y MP. Lechuga, "Evolución de la literatura sobre la responsabilidad social en pymes como disciplina científica", Revista Europea de Dirección y Economía de la Empresa, pp. 117-128. 2015.

3. D. Jiménez., y I. Pérez, "La representación simbólica de la responsabilidad social empresarial (RSE): el Caso Santa Marta", Investigación e Innovación en Ingenierías, Vol. 4, nº.2, 2016.

4. AL. Salazar, J. Ojeda., y M. Ríos, "La responsabilidad social empresarial desde la percepción del capital", Revista de Contabilidad - Spanish Accounting Review, pp. 1-11, 2017.

5. M. Lozano, L. Albareda., y T. Ysa, “¿Qué pueden hacer los gobiernos para promover la Responsabilidad Social de la Empresa (RSE)?", Revista de Economía Pública, Social y Cooperativa, vol. 13, 2005.

6. M. Tella, "Los "dones" del marketing social. AlBR", Revista de Antropología Iberoamericana, vol. 39, 2005.

7. CE. Torres, "Aportes a la responsabilidad social", Revista Mexicana de Ciencias Políticas y Sociales, pp. 393-407, 2017.

8. G. Silvestri, "La pampa y el río. Una hipótesis de registros y periodizaciones en el paisaje rioplatense", Materia, vol. 2, pp. 75-96, 2002.

9. J. Harley, La nueva naturaleza de los mapas. Ensayos sobre la historia de la cartografía. México: FCE, 2005.

10. H. Gadamer, Verdad y Método I y II. España: Sígueme Ediciones, 1999.

11. P. Ricoeur, Del texto a la acción. Ensayos de hermenéutica II. México: Fondo de Cultura Económica, 2002.

12. A. Núñez, E. Aliste, y A. , "Patagonia-Aysén en la construcción del imaginario geográfico de la nación", Itzapalapa, Revista de Ciencias Sociales y Humanidades, vol. 76, pp. 165-188, 2014.

13. G. Vargas, "Constitución del sujeto y constitución subjetiva del mundo", Revista Colombiana de Educación, n. 50, pp. 163-176, 2006.

14. R. Hernández, C. Fernández, y P. Baptista, Metodología de la investigación. México: Mc. Graw - Hill., 2014.

15. J. Aguirre., y L. Jaramillo, "Consideraciones acerca de la investigación en el aula: más allá de estar a la moda", Educación y Educadores, vol. 11, n. 1, pp. 43-54, 2008.

16. E. Guedes, "Mapeo de actores sociales: un enfoque de redes sociales en el marco del desarrollo local", Universidad de la República, Uruguay., 2004. 
17. A. López, G. Pérez., y M. Gutiérrez, "La I-A-P con los vecinos", en VV. AA "Métodos de Investigación Social", Cuadernos de la RED, 3; Red CIMS., 1995.

18. J. Larrán, M. Lechuga, D. Matínez, y J. Madueño, "Responsabilidad social en las pymes: análisis exploratorio de factores explicativos", Revista de Contabilidad, pp.1-14, 2016.

19. A. Pulido, "Desarrollo sostenible: un reto central para el pensamiento económico", Estudios de Economía Aplicada, vol. 21-2, pp. 203- 220., 2003. 